\title{
A New Buffer and Energy Based Scheduling Scheme for Supporting QoS in MANETs
}

\author{
Mohamed Brahma, Abdelhafid Abouaissa, and Pascal Lorenz
}

\begin{abstract}
Today, the original IEEE 802.11 standard has several problems in providing Quality of Service in MANETs. A single FIFO queue is used in best effort manner and it does not support QoS. The upcoming IEEE 802.11e was drafted to overcome these drawbacks. In this paper we describe a new multiple queuing system with an adaptive scheduling taking into account the states of buffers and energy consumption in a mobile ad hoc network. The proposed scheduling scheme uses dynamic weights for each queue. We study the performance of this scheme and compare it with the original IEEE 802.11b and the upcoming IEEE 802.11e. We show through simulations that the proposed buffer and energy based scheduling scheme improves overall end-to-end throughput, and gives better results than the original 802.11b and the Enhanced Distributed Coordination Function (EDCF), in terms of delay and total received and lost packets, as well as support service differentiation over multi-hop ad hoc networks.
\end{abstract}

Index terms: MANETs, QoS, energy, buffer, scheduling.

\section{INTRODUCTION}

A MANET for Mobile Ad hoc Network[1][2] is a packet radio network that can be considered as an autonomous distributed system composed by a set of identical mobile nodes that move independently and freely. Each node communicates with other nodes that reside within its transmission range, over relatively bandwidth-constrained radio links. Nodes cooperate in routing and packet forwarding tasks, achieving in this way a dynamic multi-hop network.

MANETs are useful in many applications because they do not need any infrastructure support. Sensor networks, disaster recovery, rescue and automated battlefields are examples of application environments. And without any centralized administration, nodes are free to move randomly and organize themselves arbitrarily. Thus, the topology may change rapidly and unpredictably.

In general, the network is expected to guarantee a set of measurable prespecified service attributes to the users in terms of end-to-end performance, such as bandwidth, delay, packet loss and jitter. But, actually with the evolution of multimedia technologies, Quality of Service (QoS) in MANETs becomes an area of great interest.

Manuscript received January 30, 2006 and revised May 04, 2006. This paper was presented in part at the Advanced International Conference on Telecommunications (AICT'06) 2006.

Authors are with the University of Haute Alsace, 34 Rue du Grillenbreit, 68008 Colmar France, \{e-mail: mohamed.brahma; abdelhafid.abouaissa; pascal.lorenz\}@uha.fr
One way to support QoS is service differentiation which can be defined as "the capability of a mechanism to differentiate between one or more classes of traffic so that packets belonging to higher priority class receive preferential treatment compared to the lower priority packets" [3]. In ad hoc networks, the current version of the IEEE 802.11 Wireless LAN standard [12] is considered as an extension to the Ethernet, which has a single First-In-First-Out queue (FIFO) used in best-effort manner. This queue has no capability to support QoS such as bandwidth guarantee, delay and loss rate. Recently, a new standard called 802.11e has been drafting [5], to provide service differentiation at the Medium Access Control sub-layer (MAC). Many studies have evaluated this draft of the standard by both analytical evaluation, as well as simulation [6], and have demonstrated the usefulness of the proposed mechanisms in 802.11e. However, the challenge with the upcoming 802.11e lies in determining how to configure the EDCF and the HCF to provide the desired services.

In this paper we present a new buffer and energy based scheduling scheme [13], we study the performance of this scheme and we compare it to the original IEEE $802.11 \mathrm{~b}$ and the Enhanced DCF function proposed in the 802.11e.

This paper is further organized as follows. In the next section, we first briefly provide an overview of quality of service, and discuss some related works in section 3. After, we investigate some QoS limitations at the 802.11 MAC layer and we discuss the QoS features of the Enhanced DCF scheme. In section 5, we describe the proposed solution for the IEEE $802.11 \mathrm{~b}$ [8]. After we apply, evaluate performance of this scheme through a set of simulations and compare it to both Enhanced DCF and the original IEEE $802.11 \mathrm{~b}$. Finally, the paper will be concluded in section 7 .

\section{Quality Of Service}

Usually, we can define Quality of Service (QoS) as a set of service requirements that a network needs to meet while transmitting data from a source to a destination. The network needs are governed by the service requirements of end user applications. The network is expected to guarantee a set of measurable prespecified service attributes to the users in terms of end-to-end performance, such as delay, bandwidth, packet loss and jitter.

The growth of the number of hosts in the Internet leads both researchers and industrials to resolve the problem of how to support QoS, or how to support service differentiation in such 
environments. Recently, several working groups like IntServ or DiffServ try to deal with this problem for IP layer in wired networks. Furthermore, wireless networks show a more critical medium which also needs QoS support for multimedia and real-time applications, to deal with the increasing number of terminals and standards and with the nature of the wireless channel. Due to the dynamic nature of the network, it is not possible to apply QoS Management techniques to negotiate Quality between users and networks [14].

In the literature, this QoS support can be done over all the layers in the network:

- QoS models that specify an architecture in which some kinds of services could be provided. It is the system goal that has to be implemented.

- QoS adaptation, which can hide all environment-related features from awareness of the multimedia-application above and provides an interface for applications to interact with QoS control.

- QoS signaling acts above the network layer, as a control center in QoS support. The QoS model determines the functionality of QoS signaling.

- QoS routing that represent a part of the network layer and searches for a path with enough resources but does not reserve resources.

- QoS MAC protocols, which are essential components in QoS ad hoc networks. QoS supporting components at upper layers, such as QoS signaling or QoS routing assume the existence of a MAC protocol, which solves the problems of medium contention, supports reliable communication, and provides resource reservation.

\section{RELATED WORKS}

In general, in an ad hoc network which consists of set of nodes that communicate with each other over multiple wireless hops, the topology may change rapidly and unpredictably over time, due to nodes mobility. These nodes collaborate to forward traffic. Since the participating nodes are usually mobile, power conservation is an important aspect to extending the lifetime of an ad hoc network. Actually, several schemes have been proposed to provide quality of service for multi-hop communication in ad hoc networks. In these schemes, the evaluation of quality of service is done in terms of end-to-end delay, packet loss rates and routing message overhead. However, since nodes in ad hoc networks are battery-powered and use a bandwidth-constrained channel to communicate, the energy consumption and the buffer management are also important metrics for this type of network.

A large amount of work on service differentiation has been carried out, especially via distributed and adaptive mechanisms, as it is the case in [9], [10], [11] and [18]. A first attempt to analytically model these mechanisms appears in [9]: the proposed model is very simple though the memoryless assumption does allow accounting for exponential backoff details. In [10] authors discuss the problem of supporting distributed admission control rule on top of an enhanced version of DCF (Distributed Coordination Function), via the definition of virtual MAC algorithm, that passively monitors the radio channel and estimates the service levels available, plus a virtual source algorithm. In addition, service differentiation can be provided in contention-based MANETs, by controlling the behavior of the backoff algorithm. Currently, the IEEE working Group defines enhancements to the QoS 802.11 MAC described bellow and called 802.11e [5][7]. In this scheme, a new access method called Hybrid Coordination Function (HCF) is introduced, which combines functions from DCF and PCF (Point Coordination Function) mechanisms. On the other hand, Enhanced Distributed Coordination Function (EDCF) is a contention-based HCF channel access specified for IEEE 802.11e. This function provides the capability for up to eight types of traffic classes. EDCF assigns a short CW (Contention Window) to high priority classes in order to ensure that in most cases, the high priority queues will be able to transmit before the low-priority ones. Indeed, different values of the CWmin are assigned for different priority classes, where high priority classes have smaller values of CWmin. Also, for further differentiation, in 802.11e different values of IFS (InterFrame Space) can be used according to traffic classes. Instead of DIFS (Distributed InterFrame Space), another Arbitration IFS (AIFS) is used. For a given class, the AIFS value should be a DIFS plus some time slots [7]. In this manner, classes with the smallest AIFS will have the highest priority. Within each mobile node, many "Traffic Categories" (TC) are defined. These traffic categories behave like virtual stations: in this case the collision rate increases very fast when the contentions to access the medium are very high, which significantly affects the goodput, the latency and thus, decreases the performance of delay-bounded traffic. Note that all these works propose solutions to differentiate services by using different priorities to access to the radio channel. In the proposed buffer and energy based scheduling scheme, instead of the priority to access to radio channel, we provide a service differentiation mechanism to handle packets over a multi-class queuing system in the layer 2. In addition, in this case a scheduler is used to service differently existing queues in each node. In this paper, we propose a new scheme based on dynamic weights. Each queue is accorded a weight dynamically calculated depending on the queue occupancy and the energy available in this node.

\section{Some Qos Limitations Of The IEeE 802.11}

In general, best effort service provided by the original IEEE 802.11 can be appropriate for data traffic. However, and unlike data, multimedia applications require guaranteed maximum end-to-end delay and guaranteed low loss rate and high bandwidth. Wireless medium has specific characteristics such as high loss rates, high latency and jitter. Indeed, providing QoS in MAC layer for applications of upper layers represents one of the most challenging tasks. One way to characterize quality of service is the prioritized QoS, which is expressed in terms of relative delivery priority, without strict and quantitative service support.

\section{A. QoS Limitations of DCF and PCF Functions}

In Distributed Coordination Function DCF defined in the original IEEE 802.11 MAC, a single First-In-First-Out (FIFO) queue is used in best-effort manner. This single queue has no capability to support quality of service. However, in timebounded multimedia applications such as videoconferencing 
or VoIP, users require a certain QoS in terms of bandwidth, delay and jitter guarantees. With DCF, all nodes compete for the resources and the access to channel. But with the same priorities, and no service differentiation is provided for high priority flows or real-time multimedia applications. In this case, throughput degradation and high delay are caused by the increasing time used by nodes for channel access contention especially at high loads.

In the other hand, when PCF has been designed to support applications requiring some QoS such as time-bounded multimedia applications, this function has some problems that lead to poor quality of service performance:

- PCF defines only a single-class round-robin scheduler, without taking into account the QoS requirements of different types of traffic.

- All the communications have to go through an Access Point (AP). This mechanism degrades the bandwidth performance of the wireless LAN.

- This function (PCF) can not control the transmission time of a polled station (STA). A polled STA is allowed to send a frame (length between 0 and 2304 bytes), which can introduce variable transmission time. Thus, the AP in this case can not predict exactly transmission time, which prevents the AP from providing guaranteed delay and jitter performance for nodes in the polling list during the rest of the Contention Free Period (CFP) interval.

\section{B. 802.11e: Enhanced DCF for QoS Support in WLANs}

The upcoming 802.11e's Enhanced DCF (EDCF) uses priority to provide different service levels to each Traffic Category. The goal of this new function is to enhance current DCF mechanisms to support service differentiation. EDCF provides the capability for up to eight types of traffic classes (8 queues) in each mobile station. Each quality of service station (QSTA) may have up to 4 access categories (AC), which means 4 queues at MAC layer to support 8 different priorities. Each TC maintains specific parameters: Arbitration InterFrame Space AIFS[TC], CWmin[TC] and Persistence Factor PF[TC]. Each station supports different backoff instances parameterized with TC specific parameters.

In Fig. 1 we can see a comparison between the 802.11e architecture that supports queue-based differentiation with the original single-queue based DCF access mechanism.

In the 802.11e EDCF, new parameters have been introduced:

- Contention window limits, which are used to compute the random backoff. These values: CWmin and CWmax are not fixed, as with DCF but are variable. CWmin[TC] and $\mathrm{CWmax}[\mathrm{TC}]$ values depend on the priority of the traffic.

- AIFS: the minimum specified idle duration time is not a constant value (DIFS) as in DCF, but is a distinct value AIFS[TC].

- PF: this factor has been proposed in previous version of the $802.11 \mathrm{e}$ draft, but it has been removed later [12]. It determines how quickly the $\mathrm{CW}$ is increased after a collision (i.e. high priority traffics will have the smallest PF value).

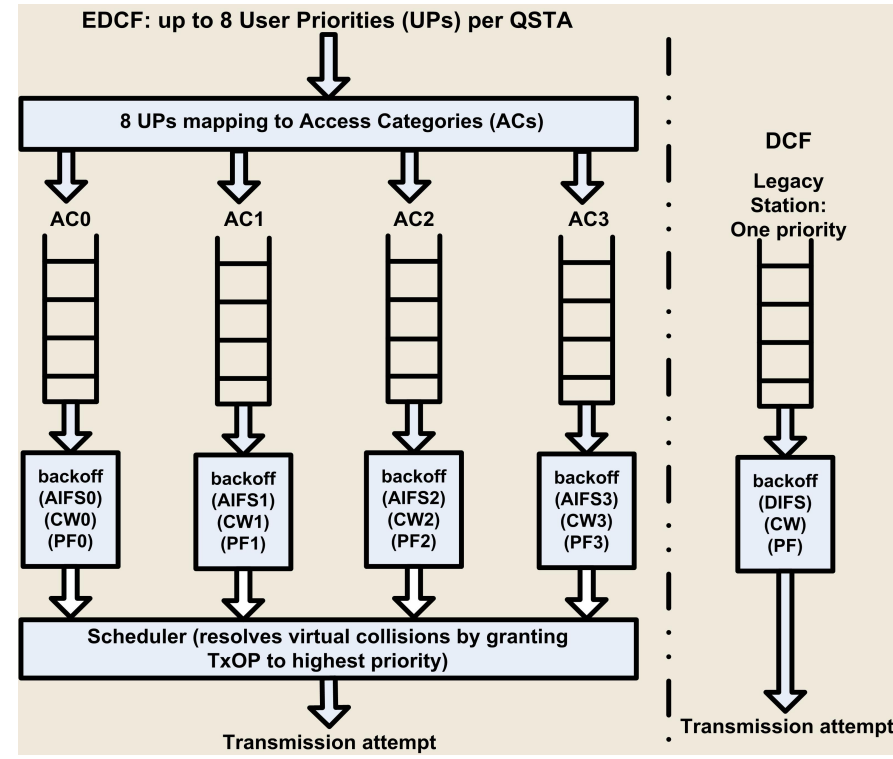

Fig. 1. Queue-based EDCF vs. basic DCF.

- TXOP (Transmission Opportunity): [16][17] the time interval permitted for a particular station to transmit packets.

Service differentiation in Enhanced DCF is provided by using different $\mathrm{CWmin}$ and different inter-frame space values (Fig. 2 shows the different relationships between the different inter-frame spaces) for each backoff instance corresponding to certain priority class. Thus higher priority traffics will get more transmission time than lower priority traffics. And the contention window value of a traffic category is given by the following equation:

$$
\text { New CW[TC }]=(\text { old CW[TC }]+1) * \text { PF }[\mathrm{TC}]-1
$$

Remark: In EDCF, all collisions between contending queues within a node are resolved within this node such that the higher priority queue receives the transmission opportunity (TXOP) and the lower priority colliding queues behave as if there were an external collision on the medium. This explains why the medium utilization of EDCF is comparably low since much time is wasted on collisions, which impacts the performance, especially at high loads.

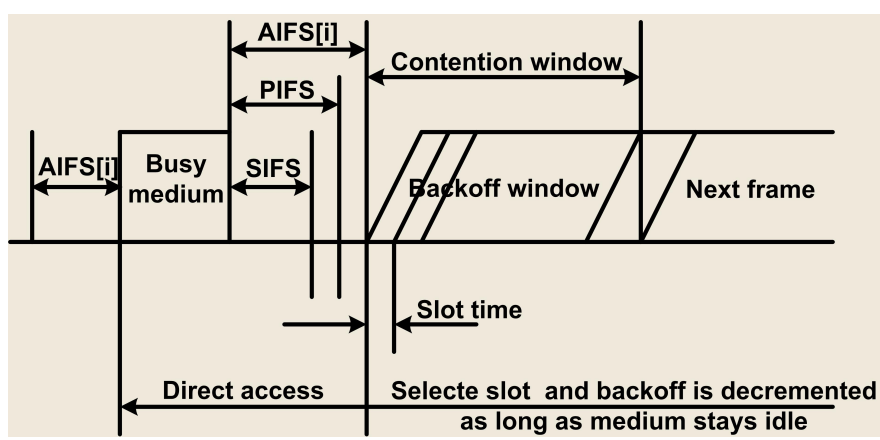

Fig. 2. IFS relationships. 


\section{The Proposed MeChanism}

Usually, in an ad hoc network, each mobile node can serve as a host as well as a router and uses consequently its own resources (energy, buffers ...) to convey packets intended for other mobile nodes in the network. Mobile nodes have in general a finite battery capacity. Moreover, in many cases they are installed in an environment where it may be difficult and undesirable to retrieve batteries to change or recharge them. In fact, it is very important to design some techniques to reduce the energy consumption in MANETs. In the other hand, and as it was presented above, the original $802.11 \mathrm{~b}$ based DCF access mechanism uses a single First-In-First-Out (FIFO) queue used in best-effort manner. In this paper, we present a multipleclass queuing system using an adaptive Weighted Round Robin (WRR) scheme, which takes into account battery life and load of high priority traffic to provide service differentiation [13].

As actual energy of the battery becomes low and/or high priority queue becomes full, the proposed mechanism gives more weight to high priority queue. So high priority flows would get more chance to be served when energy becomes lower and number of high priority packets in the queue becomes higher.

The proposed scheme consists as shown in Fig. 3 of:

Two class queues: to simplify the mechanism, we define two classes of traffic; low priority class for best effort traffic and high priority class for multimedia traffic.

Scheduler: using dynamic weights for queues.

Forwarding module: an important design goal of the proposed system is to differentiate mobile nodes acting as routers and those acting as hosts. In this case, the packet forwarding function is performed in layer 2, and it will not be necessary to handle forwarding packets in layer 3 . Hence, the power consumption and the cost are reduced.

Battery measurement module: this module is monitoring actual energy of the battery and reports it to the scheduler.

\section{- IP-MAC802.11 mapper.}

Weighted Round Robin (WRR) is a best-effort scheduling algorithm. It is the simplest emulation of Generalized Processor Sharing (GPS) algorithm. While GPS serves infinitesimal amount of data from each nonempty queue, WRR serves a number of packets for each nonempty queue $($ number $=$ normalized (weight $/$ mean packet size)), and to obtain normalized set of weights a mean packet size must be known.

In the algorithm proposed in this paper, the main feature of adaptive Weighted Round Robin is to regulate the weights of high and low priority queues according to high priority traffic load and energy consumption (battery). Regulation of the weights of each queue makes it capable to guarantee (at least) a minimum throughput of best-effort traffic under severe congestion. When a packet arrives, the forwarding module makes routing decision depending on forwarding table in layer 2. After, the packet is sent to IP layer if the current node is the destination, otherwise to the classifier. Then, the classifier pushes the packet into the associated queue.

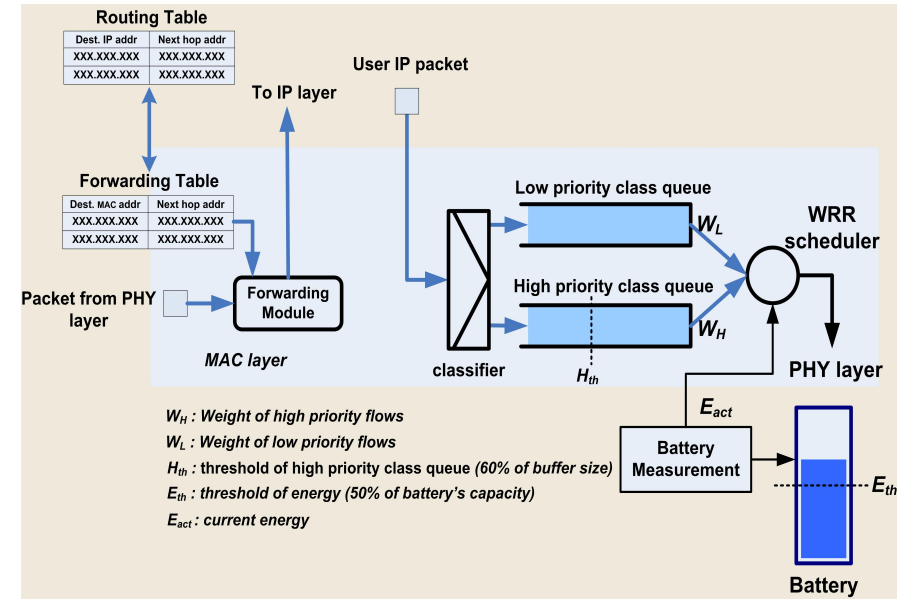

Fig. 3. Buffer and Energy Based Scheduling.

\section{A. Proposed mapping and marking 802.11 frames}

In order to give priority to MAC 802.11 packets in layer 2, we use some reserved values of sub-type in MAC header (as shown in Fig. 4). When type in MAC header is DATA (10), the Most Significant Bit (MSB) of sub-type indicates the priority of the packet. We use values from 0000 to 0111 to indicate low priority packets and values from 1000 to 1111 to indicate high priority packets.

Remark: IP-MAC802-11 mapper performs the mapping of priority between IP and MAC before encapsulate packet into MAC frame.

\section{B. Adjusting class-queues weights}

Weights of high and low priority queues are adjusted according to two parameters: the high priority traffic load and the residual energy. To adjust these weights, we used two values (thresholds) and the length of high-class queue. Each weight is defined as follows:

$$
\begin{aligned}
& W_{H}=W_{H i}+\max \left(\frac{E_{t h}-A}{E_{t h}}, \frac{B-H_{t h}}{1-H_{t h}}\right) *\left(1-W_{H i}\right) \\
& W_{L}=1-W_{H}
\end{aligned}
$$

Where $\mathrm{B}$ is the high priority queue status and $\mathbf{E}_{\mathbf{t h}}$ is the threshold for energy. These parameters are defined as follows:

$$
\mathrm{B}=\frac{\text { buffer occupancy }}{\text { buffer size }} \mathrm{A}=\frac{\text { current energy }}{\text { battery capacity }}
$$

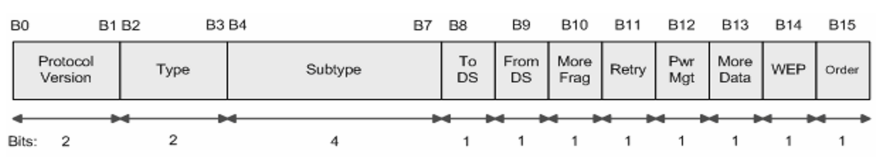

Fig. 4. Frame control format of 802.11 MAC header. 
$\mathbf{W}_{\mathbf{H}}$ represents the weight of the high priority class and $\mathbf{W}_{\mathbf{L}}$ the weight of the low priority class. $\mathbf{H}_{\text {th }}$ is the threshold of the high priority class queue. $\mathbf{W}_{\mathbf{H i}}$ is initial weight of high priority queue and $\mathbf{W}_{\mathbf{H}}$ is varying from $\mathbf{W}_{\mathbf{H i}}$ to 1.0. When $\mathbf{W}_{\mathbf{H}}$ is closed to 1.0 , only high priority packets are served. Adaptive Weighted Round Robin (WRR) scheduler chooses the queue according to its weight and transmits packet from the queue to MAC.

\section{Algorithm for adjusting dynamic weights Variables definition:}

\author{
$\mathrm{H}_{\mathrm{th}}$ : threshold of high priority buffer \\ $\mathrm{E}_{\mathrm{th}}$ : threshold of battery \\ cap: capacity of battery \\ energy $_{\text {act }}$ : current energy
}

\section{Initial Conditions:}

$$
\begin{aligned}
& W_{H i}=0.6 ; \quad W_{L i}=1-W_{H i} ; \\
& A=\text { energyact/cap } ; \quad B=\text { buffer occupancy / buffer size; }
\end{aligned}
$$

$$
\begin{aligned}
& \underline{\text { While (queues are not empty) } \underline{\text { do }}} \\
& \underline{\text { If } E_{t h}>A \text { and } B>H t h} \\
& \\
& W_{H}=W_{H i}+\left[\max \left(\left(E_{t h}-A\right) / E \text { th, }\left(B-H_{t h}\right) /\left(1-H_{t h}\right)\right)\right]^{*}\left(1-W_{H i}\right) ; \\
& / * \text { the greater variation is taken into account } * / \\
& W_{L}=1-W_{H} ;
\end{aligned}
$$

$$
\begin{aligned}
& \underline{\text { ElseIf }} E_{t h}>A \text { and } B<=H_{t h} \\
& W_{H}=W_{H i}+\left[\left(E_{t h}-A\right) / E_{t h}\right] *\left(1-W_{H i}\right) ; W_{L}=1-W_{H}
\end{aligned}
$$$$
\underline{\text { ElseIf }} \text { Eth }<=A \text { and } B>H \text { th }
$$$$
W_{H}=W_{H i}+\left[\left(B-H_{t h}\right) /\left(1-H_{t h}\right)\right] *\left(1-W_{H i}\right) ; W_{L}=1-W_{H} ;
$$$$
\underline{\text { Else }} W_{H}=W_{H i} ; W L=W_{L i}
$$

\section{Simulation Methodology And Results}

To evaluate and compare the performance of the proposed scheme with both the original IEEE 802.11 and the Enhanced DCF implemented in [6], we used the Network Simulator NS2 [15], which is a freely available discrete-event object-oriented network simulator. NS2 provides a framework for building a network model, specifying data input, analyzing data output and presenting results. The network model used for simulation is shown in Fig. 5. In the topology of the simulation, which is rather simple, we configure an ad hoc network that consists of ten mobile nodes and we define eight Constant Bit Rate (CBR) traffic sources. Each CBR source generates packets every an interval time, which is varying from an initial to a final value. The packet size is set to $1 \mathrm{~K}$ bytes.

Two traffic categories TC are defined. Half of traffic sources are high priority and rests of them are low priority. And for routing protocol, we used Ad Hoc On demand Distance Vector Routing protocol AODV [4].

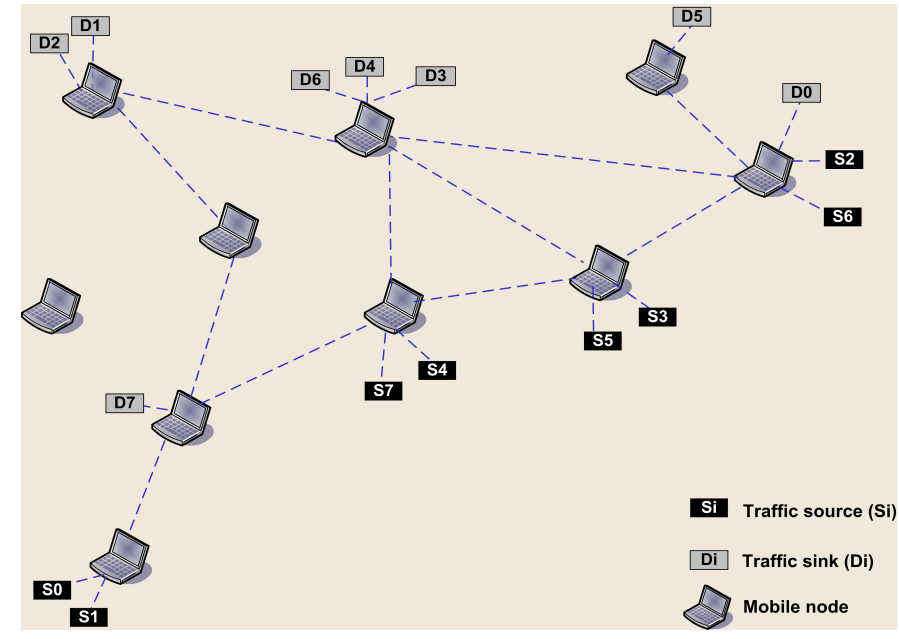

Fig. 5. Simulation model.

In order to avoid the bias of random number generation, simulation is executed 10 times for each configuration. In this network simulation model, the priority of CBR flows 1, 3, 4 and 6 are set to high and flows $0,2,5$ and 7 are set to low. We assigned 25 packets to each class queue size for the proposed queuing system and 50 packets to drop-tail queue for the comparison between the proposed scheme and the original IEEE 802.11. After, we assigned 50 packets to each class queue size for comparison with EDCF. Initially, all the nodes are assumed to have full battery of 200 joules; battery capacity was set to enough value to scale down the simulation time. Receiving and transmitting power values are set to default values as given by NS2 (see Table 1).

TABLE I

PARAMETERS Of ENERGY MOdel In Ns2

\begin{tabular}{||l||c||c||}
\hline \multicolumn{1}{|c|}{ Attribute.... } & Description & Value \\
\hline \hline -initialEnergy & $\begin{array}{c}\text { Given energy for } \\
\text { each node }\end{array}$ & 200 Joules \\
\hline \hline -rxPower & Receiving power & $281.8 \mathrm{mWatt}$ \\
\hline \hline -txPower & Transmitting power & $281.8 \mathrm{mWatt}$ \\
\hline
\end{tabular}

A. Comparing buffer and energy based scheme with original IEEE $802.11 b$

For performance evaluation of the proposed scheme, we executed simulations while varying the transmission rate from 80 to $160 \mathrm{kbps}$. Fig. 6 shows the average loss rate of low and high priority packets using both the original IEEE $802.11 \mathrm{~b}$ and the proposed scheme. As can be seen here, the percentage of packet loss rate of high priority packets is very low in the proposed scheme, compared to drop-tail queue used in the original one. However, the percentage of packet loss rate of low priority packets is little bit greater than the original scheme. This is due to the use of dynamic weights in the proposed scheme, which are accorded to both high and low priority queues: our algorithm accord (when energy is low and/or high priority queue is full) a high weight to the high priority queue, and decrease low priority queue weight. 


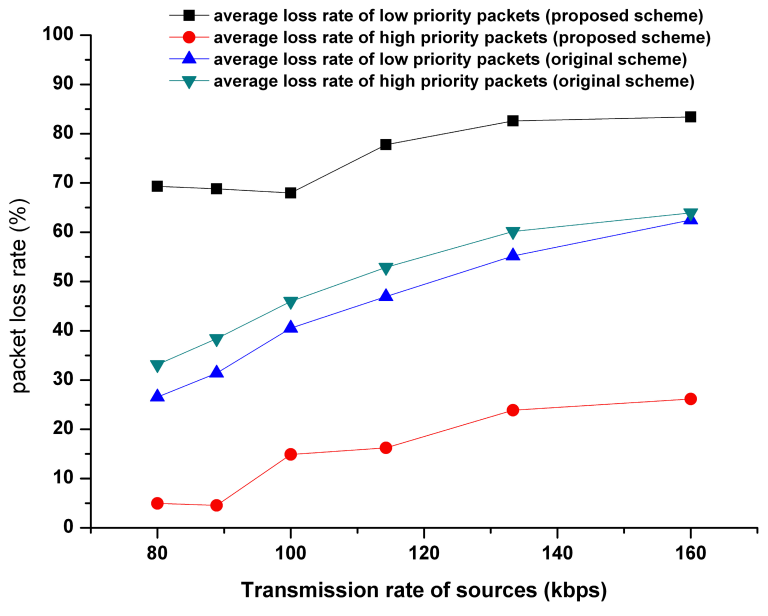

Fig. 6. The percentage of packet loss.

In Fig. 7, we see that the proposed scheme increases significantly the number of received high priority packets by an average of $130 \%$ more than the drop-tail queue. We can observe that the number of received high priority packets is increased as transmission rate of high priority $\mathrm{CBR}$ is increased. Indeed the weights of high and low priority queues are dynamically varied (as the number of packets waiting in the high priority queue is increased and/or energy is decreased, high priority packets are more served).

Fig. 8 represents the average latency of high and low priority packets using the original IEEE $802.11 \mathrm{~b}$ scheme and the proposed buffer and energy based scheduling scheme. Obviously, when traffic load is low, end-to-end latency of high priority packets keeps low. But when traffic load becomes heavy, delay also becomes slightly large. This can be achieved by the scheduler at the expense of long queuing delay of low priority packets.

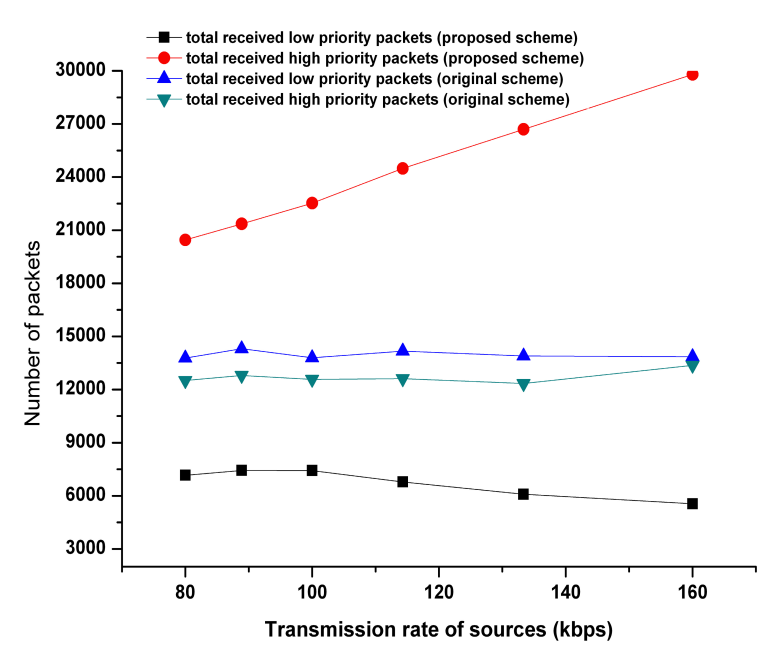

Fig. 7. The number of received high and low priority packets.

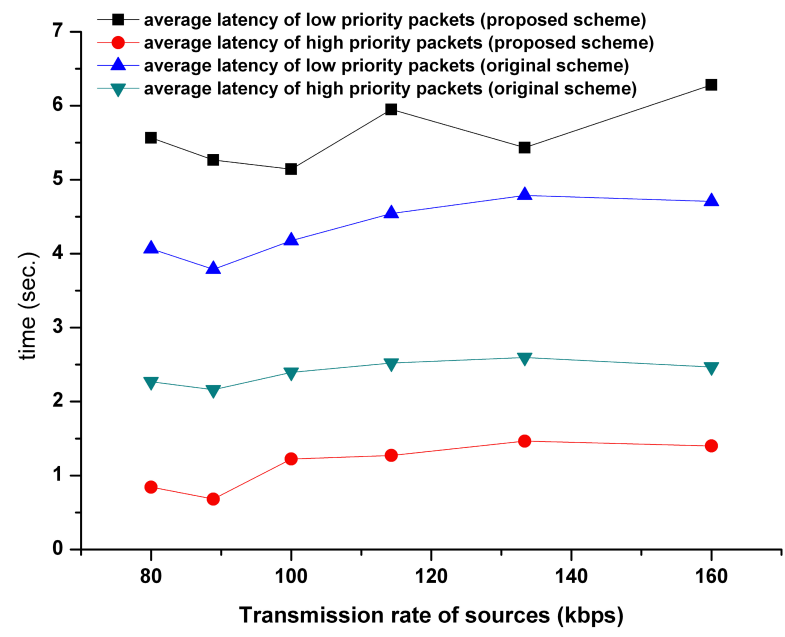

Fig. 8. The average latency.

\section{B. Comparing proposed scheme with Enhanced DCF}

For performance evaluation of the proposed scheme, we use a topology that consists of 10 mobile nodes in $1200 \mathrm{~m} \times$ $1200 \mathrm{~m}$ area. Fig. 9 plots the percentage of packet loss rate of high and low priority packets using both Enhanced DCF and buffer and energy based scheduling scheme. We can see that percentage of packet loss rate of high and low priority packets is very low in the proposed scheme, compared to the QoS Enhanced DCF. The percentage of packet loss rate of low priority packets is also lower in the proposed scheme than the EDCF one. The reduced loss rate in this case is due to the use of dynamic weights in the proposed scheme, where we accord high weights to the high priority queue depending on energy and queue occupancy. Also, the proposed scheme uses a scheduler to manage all access to the medium between queues. However, in the EDCF mechanism queues are always in competition to access the medium, which can increase the number of collisions and the packet loss rate.

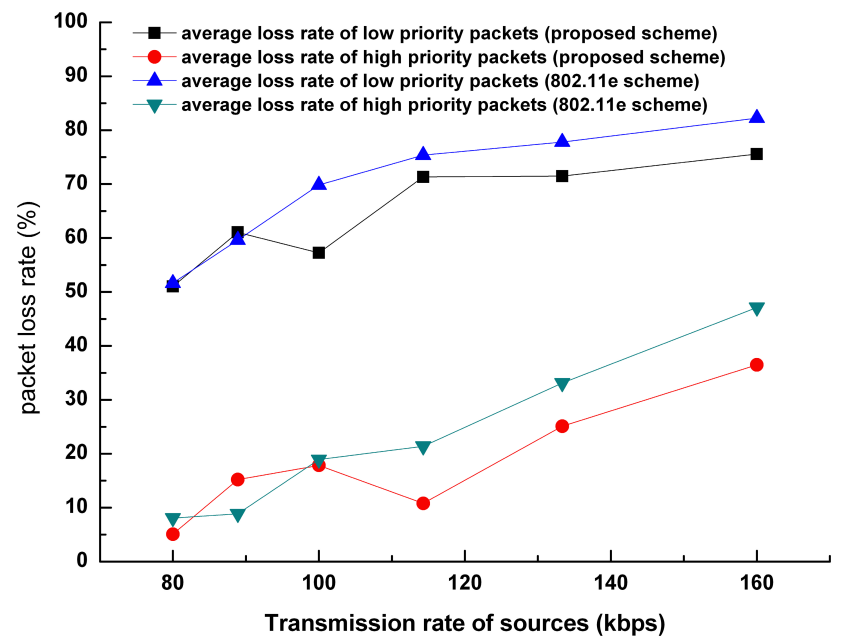

Fig. 9. The percentage of packet loss. 
Fig. 10 represents the number of received high and low priority packets using both the upcoming EDCF of $802.11 \mathrm{e}$ and the proposed scheme. We can observe that the buffer and energy based scheduling scheme enhances significantly the overall performance in terms of received high priority packets, especially when transmission rate is equal to $160 \mathrm{kbps}(26 \%$ than the EDCF). The number of received low priority packets is also little bit better than the 802.11e EDCF. These results can be explained as follows: we can see two phases in these curves: the first one is before $120 \mathrm{kbps}$ and the second one is after this value. In the first phase, for most values of transmission rate, the number of received low priority packets in the proposed mechanism is nearly same. However after this value, it decreases. In the second phase as the number of packets waiting in the high priority queue is increased (which can be justified by the increasing of transmission rates of sources) and/or energy is decreased, the proposed mechanism increases dynamically the weight of the high priority queue. In fact high priority packets will be more served.

Fig. 11 depicts the average latency of high and low priority packets of the two schemes. We observe that end-to-end delay of high priority packets depends on traffic load. And in most cases, the buffer and energy based scheme gives relatively better results than EDCF for both high and low priority packets.

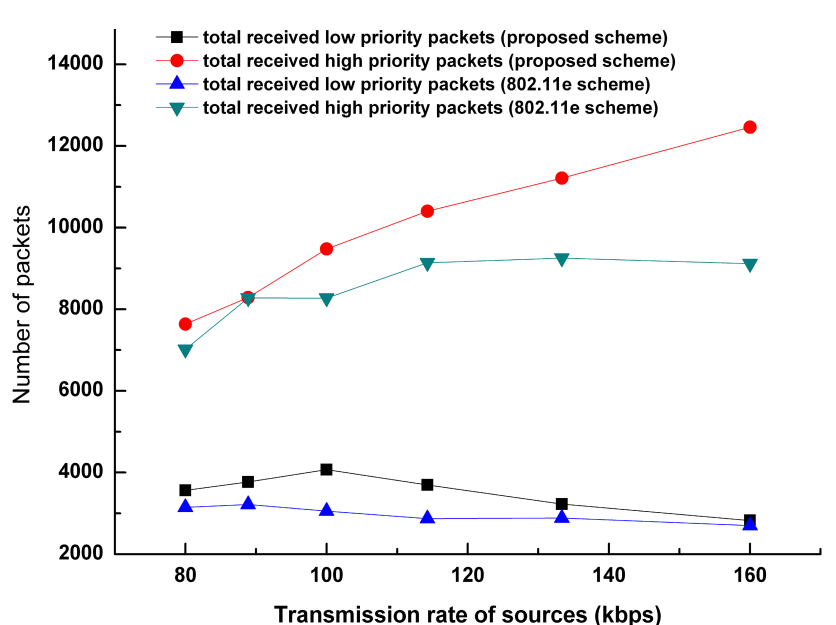

Fig. 10. The number of received high and low priority packets.

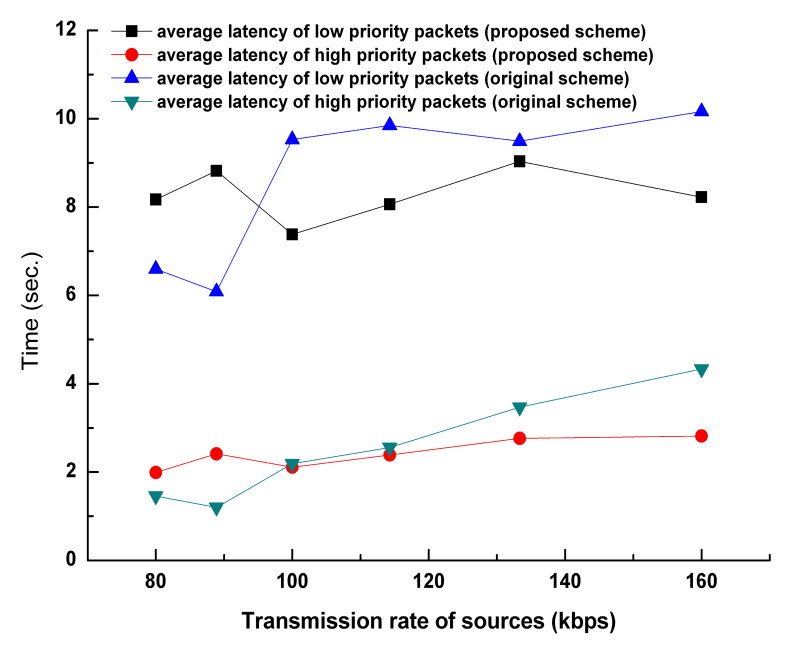

Fig. 11. The average latency.

\section{SUMMARY AND CONCLUSIONS}

In this paper we have investigated some QoS limitations at the 802.11 MAC layer. We have also examined the upcoming QoS 802.11e Enhanced DCF and presented a buffer and energy based scheduling scheme. This new scheme defines a new architecture for forwarding packets in the layer 2 and a new scheduling mechanism based on the use of multiple queuing system to support multi-classes of traffics. In the proposed scheme, Weights are dynamically accorded to each queue and are calculated using two parameters: queue occupancy and residual energy.

In order to evaluate the proposed scheme, a set of simulations have been done to compare its performance with both the original IEEE $802.11 \mathrm{~b}$ protocol and the implementation of the upcoming QoS scheme 802.11e. Simulation results show that the proposed buffer and energy based scheduling scheme improves throughput and reduces significantly the packet loss rate of high priority flows. For our future research work, we are looking for the integration of this mechanism in the QoS scheme 802.11e.

\section{REFERENCES}

[1] C. E. Perkins, "Ad Hoc Networking". Addison-Wesley, Upper Saddle River, NJ, USA, Jan 2001.

[2] I. Chlamtac, M. Conti, J.J. Liu, "Ad Hoc Networks" vol. 1, no. 1, pp. $13-64$, Jan 2003

[3] P. Ferguson, G. Huston, "Quality of Service: Delivering QoS on the Internet and in Corporate Networks". John Wiley and Sons, January 1998.

[4] E. M. Royer, C. E. Perkins, "An Implementation Study of the AODV Routing Protocol". vol. 3, pp. 1003-1008, Proceedings of the IEEE Wireless Communications and Networking Conference, Chicago IL. September 2000.

[5] IEEE WG, 802.11e Draft 3.1, May 2002.

[6] S. Wiethoelter, C. Hoene, "Design and Verification of an IEEE 802.11e EDCF Simulation Model in ns-2.26". Technical Report TKN-03-019, Telecommunication Networks Group, Technische Universitat Berlin, November 2003.

[7] L. Romdhani, Q. Ni, T. Turletti, "Adaptive EDCF: Enhanced Service Differentiation for IEEE 802.11 Wireless Ad Hoc Networks". IEEE Wireless Communications and Networking Conference (WCNC 2003), New Orleans, Louisiana, USA, March 16-20, 2003.

[8] L. Marja-Leena "IEEE 802.11 Wireless LAN". (2000). http://www.tml.hut.fi/Opinnot/Tik-10.551/2000/papers/IEEE 802 /wlan.html.

[9] I. Aad, C. Castelluccia, "Differentiation mechanisms for IEEE 802.11". Proc. of IEEE INFOCOM, pp. 209-218, (2001).

[10] M. Barry, A. T. Campbell, A. Veres, "Distributed Control Algorithms for Service Differentiation in Wireless Packet Networks". Proc. of IEEE INFOCOM 2001, pp. 582-590.

[11] G. Bianchi, I. Tinnirello, "Analysis of Priority Mechanisms based on Differentiated Inter-Frame Spaces in CSMA/CA". (2003). in Proc. IEEE VTC 2003, vol. 3, pp. 1401 - 1405, Orlando (FL).

[12] IEEE 802.11 WG, IEEE Std 802.11e/D8.0, Draft Supplement to IEEE standard for Telecommunications and Information exchange between systems. "Local and Metropolitan Area Networks". Specific Requirements Part 11: Wireless LAN Medium Access Control (MAC) and Physical Layer (PHY) specifications. February 2004. 
[13] M. Brahma, K.W. Kim, A. Abouaissa, P. Lorenz, "A Buffer and Energy Based Scheduling in Mobile Ad hoc Networks over Link Layer". The Advanced International Conference on Telecommunications AICT'06. Guadeloupe, French Caribbean, February 19-22, 2006.

[14] M. Barry, S. McGrath, "QoS Techniques in Ad Hoc Networks". (2003). Proceedings of 1st International ANWIRE Workshop. Glasgow, Scotland.

[15] Internet homepage of the NS2: http://www.isi.edu/nsnam/ns.

[16] S. Choi et al., "Multiple Frame Exchanges during EDCF TXOP", IEEE 802.11-01/566r3, January. 2002.

[17] S. Choi et al., "IEEE 802.11e Contention-Based Channel Access (EDCF) Performance Evaluation", in Proc. IEEE ICC'03, May 2003.

[18] A. Ksentini, M. Naimi, A. Nafaa and M. gueroui, "Adaptive service differentiation for QoS provisioning in IEEE 802.11 wireless ad hoc networks", Proceedings of the 1st ACM international workshop on Performance evaluation of wireless ad hoc, sensor, and ubiquitous networks, Venezia, Italy, Pages: $39-45$ (2004)

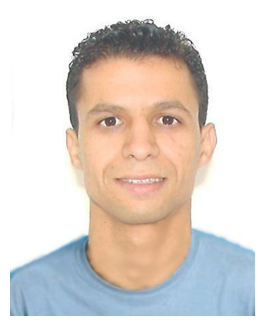

Mohamed BRAHMA received the Master degrees in Distributed Systems and Networking from Franche-Comté University, France in 2003. He is currently a $\mathrm{PhD}$ student at the Network and Telecommunication Research Group at the University of Haute Alsace, France. His research interests include mobile wireless networks, QoS support, resource management in ad hoc networks and distributed systems.

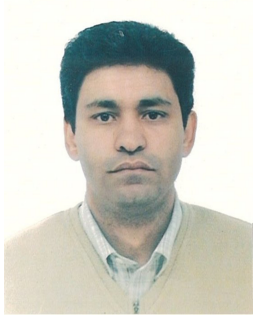

Abdelhafid Abouaissa is an Associate Professor at the University of Haut Alsace, in Colmar France. He received the BS degree from Technical University of Wroclaw, Poland, in 1995, and the MS degree from Franche Comte University of Besançon, France, in 1996. He obtained the $\mathrm{PhD}$ at Technical University of Belfort, France in January 2000. His interests include multimedia synchronization, group communication systems, Ad-Hoc, MPLS, DiffServ, and QoS management.

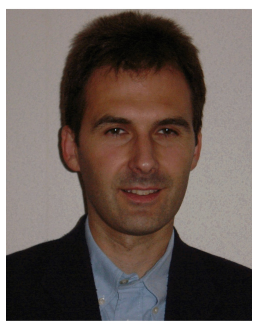

Pascal Lorenz received the $\mathrm{PhD}$ degree from the University of Nancy, France. Between 1990 and 1995 he was a Research Engineer at WorldFIP Europe and at Alcatel-Alsthom. He is a Professor at the University of Haute-Alsace and is responsible for the Network and Telecommunication Research Group. His research interests include QoS, wireless networks, and high-speed networks. He was the Program and Organizing Chair of the IEEE ICATM'98, ICATM'99, ECUMN'00, ICN'01, ECUMN'02 and ICT'03, ICN'04 conferences and Co-Program Chair of ICC'04. Since 2000, he has been a Technical Editor of the IEEE Communications Magazine Editorial Board. He is the Secretary of the IEEE ComSoc Communications Systems Integration and Modelling Technical Committee. He is a Senior Member of IEEE, a member of many international program committees and has served as a guest editor for a number of journals including Telecommunications Systems, IEEE Communications Magazine and LNCS. He has organized and chaired several technical sessions and given tutorials at major international conferences. He is the author of 3 books and 135 international publications in journals and at conferences. 